
and Frank Meyer

\title{
Unsupervised and weakly supervised approaches for answer selection tasks with scarce annotations
}

https://doi.org/10.1515/comp-2019-0008

Received February 20, 2019; accepted June 25, 2019

\begin{abstract}
Addressing Answer Selection (AS) tasks with complex neural networks typically requires a large amount of annotated data to increase the accuracy of the models. In this work, we are interested in simple models that can potentially give good performance on datasets with no or few annotations. First, we propose new unsupervised baselines that leverage distributed word and sentence representations. Second, we compare the ability of our neural architectures to learn from few annotated examples in a weakly supervised scheme and we demonstrate how these methods can benefit from a pre-training on an external dataset. With an emphasis on results reproducibility, we show that our simple methods can reach or approach state-of-the-art performances on four common AS datasets.
\end{abstract}

Keywords: neural networks; natural language processing; question answering; answer selection

\section{Introduction}

Large-scale Question Answering (QA) tasks have recently received a substantial amount of attention. In this paper, we are interested in the QA subtask of Answer Selection (AS), where the goal is to retrieve the correct answers to a question amongst a set of candidate answers. While a vari-

\footnotetext{
^Corresponding Author: Emmanuel Vallee: Orange Labs, Paris, France, E-mail: manu.vallee@gmail.com

Delphine Charlet: Orange Labs, Paris, France,

E-mail: delphine.charlet@orange.com

Francesca Galassi: INRIA, Paris, France,

E-mail: francesca.galassi@inria.fr

Gabriel Marzinotto: Aix Marseille Univ, Paris, France,

E-mail: gabriel.marzinotto@lis-lab.fr

Fabrice Clérot: Orange Labs, Paris, France,

E-mail: fabrice.clerot@orange.com

Frank Meyer: Orange Labs, Paris, France,

E-mail: franck.meyer@orange.com
}

ety of AS datasets exists, it appears that these are typically smaller than open domain Question Answering corpus such as SQuAD [1]. Most efforts to adress the AS task are directed towards supervised end-to-end training of complex neural architectures. Existing models are typically based on convolutional [2] or recurrent neural networks [3], with hundred of thousands of parameters, and a set of hyperparameters typically tuned for the target dataset. Additionally, they require a large amount of annotated data to perform end-to-end training. Here, we question the real added value of deep learning methods compared to unsupervised baselines and simple neural networks approaches.

The AS problem can be formalized as follows. First, the question and answer sequences are encoded via word embedding and further processing through a neural network based on convolutional [2] or recurrent [3] layers, with the possible addition of attention mechanism [4]. Second, the vectors encoding question and answer are used to predict the probability, or matching score, for the answer to be correct for the given question. Recently, it was proposed to address the AS task with simple models based on word embedding pooling operations [5] and simple neural architecture [6]. In an effort to design fully unsupervised approaches, a strong world alignment baseline was proposed in [7]. Our work takes inspiration from these simple approaches, and we aim our methods to be as simple and generic as possible. This is in opposition with most of state-of-the-art methods that rely on convolution and recurrent layers with the addition of attention mechanisms [8-10].

The main contribution of our work is addressing the issue of few or no annotated data. For this, we propose a novel unsupervised method relying on the Hungarian method [11], as well as weakly supervised neural approaches. We compare two model architectures, one that is learning the question/answer similarity, and another that is learning the question and answer vector representation before computing a cosine similarity.

To overcome the scarcity of annotated data, we propose to exploit distributed word representations, such as word2vec (w2v) [12], as well as a pre-trained sentence encoding model, the Universal Sentence Encoder (USE) [13]. 
Going one step further, we present a domain adaptation approach, where we pre-train our models on a large external QA dataset and fine-tune the models on the target dataset, similar to the work by Min et al. [9].

We assess the behaviour of our model when varying the amount of training data and observe the ability of some architecture to achieve low-shot learning. Finally, we show that our methods can reach approach state-of-the-art performance when all the training data is used.

Importantly, we make a major effort to have fully reproducible results from the initial dataset, as it is established that the deep learning community is affected by a reproducibility crisis due to poorly controlled environment settings [14].

We demonstrate on four publicly available datasets that our methods reach near state-of-the-art results. The source code to reproduce our experiments is available ${ }^{1}$.

\section{Methods}

The goal of our study is to propose guidelines over several methods addressing the AS tasks. Therefore, we present a collection of approaches applicable in different contexts, depending on the availability of annotated data and potential external resources.

\subsection{Unsupervised approaches}

The unsupervised approaches proposed in this section aim to reach acceptable performances in the case where there are no annotations available for training. First, we propose two methods relying on pre-trained word embeddings that associate a vector for each word in the vocabulary [12]. Second, we proposed to leverage a pre-trained language model, the Universal Sentence Encoder [13]. The model relies on attention modules (Transformer [15]) to encode the input sentence into a 512-dimensional vector. Finally, we compare our approaches to the BM25 information retrieval baseline [16].

BM25: The score for each candidate answer is given by the
BM25 weights of question words in the answer:

$$
\begin{aligned}
\operatorname{score}(Q, A)= & \sum_{i=1}^{n}\left(\operatorname{IDF}\left(Q_{i}\right) .\right. \\
& \left.\frac{f\left(Q_{i}, A\right) \cdot\left(k_{1}+1\right)}{f\left(Q_{i}, A\right)+k_{1} \cdot\left(1-b+b \frac{|A|}{\text { avgdl }}\right)}\right),
\end{aligned}
$$

where $\operatorname{IDF}\left(Q_{i}\right)$ is the inverse document frequency of the word $Q_{i}, f\left(Q_{i}, A\right)$ is the frequency of word $Q_{i}$ in the candidate answer $A,|A|$ is the number of words in the candidate answer, avgdl is the average number of words in the answers, $k_{1}$ is a fixed parameter with value 1.5 , and $b$ is a fixed parameter with value 0.75 .

Average word embedding: Each word is represented by a 300 dimensional vector and the word vectors are averaged across all the sentence to obtain the sentence vector. The score is given by the cosine similarity between the question and answer vectors:

$$
\operatorname{score}(Q, A)=\frac{\overline{Q_{w 2 v}} \cdot \overline{A_{w 2 v}}}{\left\|\overline{Q_{w 2 v}}\right\||| \mid \overline{A_{w 2 v}} \|},
$$

where $\overline{Q_{w 2 v}}$ and $\overline{A_{w 2 v}}$ are the word embeddings average across, respectively, all the question and answer words.

Hungarian method for embedding alignment: We compute a similarity matrix between each vector representing the words of the question and answer. We then adapt the Hungarian method [11], originally designed for minimal cost assignment, to maximal similarity assignment. The adaptation is simply performed by transforming the similarity into a cost $C_{i, j}=\max (S)-S_{i, j}$, where $C$ is the cost matrix, $S$ is the similarity matrix and $i, j$ are the $i^{\text {th }}$ question word and the $j^{\text {th }}$ answer word. The score is given by the Hadamard product between the cost matrix $C$ and the optimal alignment matrix $X$ :

$$
\begin{gathered}
\operatorname{score}(Q, A)=C \odot X \\
C_{i, j}=\max (S)-S_{i, j} \\
S_{i, j}=Q_{i} \cdot A_{j},
\end{gathered}
$$

where $Q_{i}$ and $A_{j}$ are the embedding vectors of the $i^{\text {th }}$ question word and the $j^{\text {th }}$ answer word, respectively.

USE: We encode each question and answer with the Universal Sentence Encoder (USE) [13] into a 512 dimensional vector and compute the score with the cosine similarity between the two vectors.

\subsection{Neural approaches}

Amongst the broad family of neural architectures and training approaches available in the AS literature [17], 
we propose to evaluate two basic neural architectures coupled with two learning approaches: the siamese and Concat architectures, and the point-wise and pair-wise training approaches.

\section{Architecture}

The siamese architecture learns a projection of the input vectors. It consists in one hidden layer per question and answer vector representation with shared weights, followed by a cosine similarity that gives the matching score for the Q/A pair. This architecture is of particular interest because the question and answer are represented independently. It results that a large set of candidate answers could be encoded offline. That would provide a very fast and powerful way (cosine operation) to perform a semantic match with a query encoded "on-the-fly".

The model can be written as:

$$
\begin{gathered}
h_{Q}=\operatorname{ReL} U\left(W^{h} Q+b^{h}\right) \\
h_{A}=\operatorname{ReL} U\left(W^{h} A+b^{h}\right) \\
o=\cos \left(h_{Q}, h_{A}\right),
\end{gathered}
$$

where $h_{Q}$ and $h_{A}$ are the hidden layers for the question $Q$ and the answer $A$ and $W^{h}$ are the shared weights; $o$ is the output layer, which is simply a cosine between the two vectors $h_{Q}$ and $h_{A}$.

The concat architecture learns the similarity between the question and the candidate answer. It concatenates the question and answer vectors, as well as their element-wise product and their absolute difference, to obtain the QA pair representation, written as $(Q, A, Q \odot A,|Q-A|)$. It is followed by a single hidden layer, and by a sigmoid output layer that gives the matching score for the Q/A pair. The model can be written as:

$$
\begin{gathered}
i=(Q, A, Q \odot A,|Q-A|) \\
h=\operatorname{ReLU}\left(W^{h} i+b^{h}\right) \\
o=\sigma\left(W^{o} h+b^{o}\right),
\end{gathered}
$$

where $i$ is the concatenation layer, $h$ is the hidden layer and $o$ is the output layer with a sigmoid activation that computes the probability of the answer to be relevant.

\section{Training approach}

Additionally, we compare two training approaches, where we vary the loss function. The first and simplest approach is called point-wise, where the AS problem is transformed to a binary classification problem. The classifier is trained to associate a value comprised between 0 and 1 to each candidate answer for a given question. In this case, the loss function is the binary cross-entropy.

The pair-wise approach learns to minimize the distance between the positive answer and the question while maximizing the distance between the negative answer and the question. In this case, the loss function is the triplet-loss: $\mathcal{L}=\max \left(d\left(Q, A^{+}\right)-d\left(Q, A^{-}\right)+m, 0\right)$, where $d$ is the distance between the pair given by the output layer of a given network, $Q$ is the question, $A^{+}$is a positive answer, $A^{-}$is a negative answer, and $m$ is the constant margin.

Finally, we use two different vector representations for the input sequence: the word2vec (w2v) word embedding averaged across all the sentence words, and the sentence embedding computed with the Universal Sentence Encoder (USE) [13].

\section{Domain adaptation}

To deal with the scarce annotation of our target datasets, we explore how pre-training our models on an external dataset with the same task could improve the performance. This well-known approach is what we call here domain adaptation. Following a recent study on domain adaptation for AS task [9], we pre-train our models on an external dataset and fine-tune them on the target dataset. Importantly, pre-training a model on an external dataset allows deploying the model as such, without further finetuning on the target dataset. This approach could be of major interest when no annotated data is available.

\section{Experiments}

In our experiments, we assess the performance of our methods on several AS datasets. We simulate the experimental settings where no or few annotated data is available, as well as whether external resources or datasets are available.

\subsection{Experimental data}

We evaluate our methods on four publicly available AS datasets.

WikiQA: Open domain questions extracted from Bing requests. The answers are sentences from a Wikipedia para- 
graph. There is only one sentence per candidate answer. Following recent work, we only consider questions that have correct answers for evaluation [10].

TrecQA: Dataset created from the Text REtrieval Conference (TREC) question answering tracks (8-13) [18]. Following the literature [19] , we use the CLEAN version, where all the questions with only positive or only negative answers are removed.

Semeval - Task 3 - English subtask: Questions and answers from a life forum in Qatar [20]. Note that the candidate answers are potentially composed of several sentences.

Yahoo: 10k Questions extracted from Yahoo! L5 142k Nonfactoid questions. We use the subset and the corresponding train/dev/test splits described in [21].

Importantly, Semeval and Yahoo datasets are community question answering where the questions and answers are generated by the user. As a consequence, candidate answers are complex multi-sentence texts with detailed information. The CQA datasets therefore present a domain shift with WikiQA and TrecQA, which are open domain QA with encyclopedia sourced answers.

Table 1: Datasets statistics - \#A refers to the average number of candidate answers per question, \% correct is the percentage of correct answers and Q,A length is the average number of words for the question and answers respectively.

\begin{tabular}{lccccc}
\hline Dataset & $\begin{array}{c}\text { \#Questions } \\
\text { train/dev/test }\end{array}$ & \#A $\%$ correct & Q length A length \\
\hline WikiQA & $873 / 126 / 243$ & 11 & 10.90 & 7 & 22 \\
TrecQA & $1161 / 69 / 68$ & 30 & 11.57 & 9 & 28 \\
SemEval & $1790 / 244 / 327$ & 11 & 33.78 & 48 & 35 \\
Yahoo & $5000 / 2500 / 2500$ & 10 & 9.74 & 12 & 45 \\
\hline
\end{tabular}

In Table 1, we report some basics statistics of the four datasets. We note that the training set is relatively small for all datasets, considering the size of recent question answering corpus such as SQuAD (90k training questions).

\subsection{External resources and pre-trained models}

The first external resource is the training data used to compute the word embedding. The generic $\mathrm{w} 2 \mathrm{v}$ embedding that is trained on the Google News dataset (about 100 bil- lion words) and publicly available 2 . The word2vec embedding can alternatively be trained on a large set of unannotated data from the same source as the target corpus. These specific embeddings are built from Wikipedia for WikiQA and TrecQA datasets, and from the target corpus for Yahoo answers and SemEval. We used the skip-gram w2v embedding approach with a window of 8 tokens and a minimum word count of 10.

Second, we propose to employ the pre-trained USE model to compute an embedding for the question and for the answer [13]. It produces a 512 dimensional vector for a given sentence.

Finally, we leverage a large QA dataset (SQuAD [1]) modified for sentence level AS and denoted SQuAD-T [9]. This dataset consists in 90k questions posed by crowd-workers on a set of Wikipedia articles. We note that SQuAD-T presents a domain shift with the CQA datasets, i.e., Semeval and Yahoo.

\subsection{Experimental setup}

\section{Pre-processing}

For the USE representation, no pre-processing is necessary because the USE model was trained with raw text as input. For the other methods we apply the following pipeline: switching to lower case, deleting diacritics (glyph added to a letter, or basic glyph), adding spaces around the punctuation, removing confusing signs (coma and dots in numbers, smileys), and replacing URLs by a "_url_" tag. We also remove stop-words as defined in the NLTK English list $^{3}$. We used the data format proposed in the original release of the datasets, and the pre-processing steps were the same across datasets.

\section{Networks parameters}

The hyper-parameters of our neural networks are fixed a priori and kept constant across datasets. For both architectures, the hidden layer has a number of cells equal to the dimensionality of the input layer. For example, with the siamese architecture and a USE representation $(d=512)$, the hidden layer has 512 cells, whereas with the Concat architecture, the number of cells would be $512 \times 4=2048$. Each hidden layer is followed by a dropout layer with a rate of

2 https://code.google.com/archive/p/word2vec/

3 https://www.nltk.org/ 
0.3.

The only parameter optimized across datasets is the margin for the pair-wise approach. The optimal values for the development data splits are 0.05 for SQuAD-T, 0.01 for WikiQA, 0.02 for SemEval, 0.1 for TrecQA and 0.5 for Yahoo.

The optimization is conducted with Adam and its default parameters proposed in the original paper [22]. The batch size is fixed to 32 and we use an early stopping scheme to avoid over-fitting.

\section{Evaluation}

The evaluation is performed with the official SemEval scorer to compute the metrics ${ }^{4}$. Note that we evaluate all the question sets, including when there is no correct answer for a given question.

To ensure reproducible results, each experiment is repeated 10 times with different question subsets, and we report the mean of each metric over the 10 runs.

To be consistent with the literature, we report the Mean Average Precision (MAP) for WikiQA, Semeval and TrecQA and the precision at one (P@1) for the Yahoo dataset. We consider that two results are significantly different when the $p$-value computed with the Wilcoxon test is smaller than 0.05 .

Besides performance obtained using the full training dataset, we are interested in the relationship between the amount of training data and the performance of our methods. Hence, the models are trained on the target dataset with a number of questions ranging from 4 to the full training set.

\section{Results}

\subsection{No labels on the target dataset}

In this section, we report the results obtained without any training on the target dataset, i.e., we simulate the case where no annotations are available. We present the unsupervised methods as well as the neural models trained on an external dataset and deployed on the target dataset without any further fine-tuning.

4 http://alt.qcri.org/semeval2016/task3/index.php?id=data-andtools

\section{Unsupervised methods}

In Table 2 (top and middle rows), we report the results obtained with the IR baseline and the unsupervised methods on the four datasets. We reach acceptable performance with fully unsupervised baselines, and two methods stand out from the others. The Hungarian approach with a specific word embedding performs the best on three datasets (TrecQA, SemEval and Yahoo), with a significant difference with the second best (Hungarian with generic embedding). On the Yahoo dataset, there is no significant difference between generic and specific embedding, neither with the Hungarian nor the average embedding approach.

We note that the USE representation performs best only on the WikiQA dataset, with a significant difference with the Hungarian method.

Table 2: Results obtained with fully unsupervised methods. * indicates that the best result presents a significant difference with the second best result for a given dataset.

\begin{tabular}{llllll}
\hline Method & \multicolumn{2}{c}{ Specific WikiQA } & \multicolumn{2}{c}{ TrecQA } & \multicolumn{2}{c}{ SemEval Yahoo } \\
& w2v & MAP & MAP & MAP & P@1 \\
\hline \hline wordcount & N/A & 53.83 & 57.48 & 61.32 & 15.52 \\
BM25 & N/A & 58.52 & 67.77 & 60.95 & 20.04 \\
\hline w2v cos & No & 60.22 & 56.61 & 62.91 & 23.76 \\
& Yes & 61.83 & 67.66 & 64.15 & 24.16 \\
Hungarian & No & 64.40 & 71.47 & 64.52 & $\mathbf{2 9 . 4 8}$ \\
& Yes & 65.98 & $76.16 *$ & $66.15^{*}$ & 29.08 \\
USE cos & No & $69.66^{*}$ & 72.23 & 64.16 & 22.40 \\
\hline
\end{tabular}

\section{Distantly supervised neural approaches}

In Table 3, we illustrate the results obtained with the neural models pre-trained on the SQuAD-T dataset and deployed on the target dataset without fine-tuning. We refer to this approach as distantly supervised. We show the concat architecture coupled with the point-wise training, and the siamese architecture coupled with the pair-wise training as they prove to be the best performing combinations. We also compare how the representation (w2v or USE) affects the performance.

Our first observation is that the USE representation outperforms the $\mathrm{w} 2 \mathrm{v}$ representation, except on the TrecQA dataset with the pair-wise/siamese approach. Comparing the pair-wise/siamese and point-wise/concat approaches, we observe that the first one performs better on the CQA 
dataset, while the second performs better on the QA (WikiQA and TrecQA) datasets. The difference is however smaller on the QA datasets, with respectively -0.8 and 0.01 points for WikiQA and TrecQA, compared to the pointwise/concat. As a consequence, it is safe to recommend the point-wise/concat architecture when no assumption is made on the target dataset.

Overall, the obtained performance is superior to the fully unsupervised approaches on WikiQA and Semeval, while the Hungarian method is better for TrecQA and Yahoo.

Table 3: Results obtained with distantly supervised neural approaches. The models are pre-trained on SQUAD-T, without finetuning on the target dataset. * indicates that the best result is significantly different from the second best result for a given dataset.

\begin{tabular}{llllll}
\hline Method & \multicolumn{3}{c}{ Specific WikiQA } & TrecQA & \multicolumn{2}{c}{ SemEval Yahoo } \\
& w2v & MAP & MAP & MAP & P@1 \\
\hline \hline point/concat w2v & No & 61.48 & 69.12 & 64.33 & 21.84 \\
point/concat w2v & Yes & 62.95 & 70.71 & 63.92 & 22.92 \\
point/concat USE & No & 75.70 & 72.05 & $70.16 *$ & $\mathbf{2 7 . 7 2 *}$ \\
pair/siamese w2v & No & 62.67 & 68.44 & 62.86 & 23.00 \\
pair/siamese w2v & Yes & 67.32 & $73.84 *$ & 64.33 & 23.08 \\
pair/siamese USE & No & $\mathbf{7 6 . 4 8 *}^{*}$ & 72.04 & 68.49 & 24.52 \\
\hline
\end{tabular}

\subsection{Few labels in the target dataset}

In Figure 1 and Figure 2, we show the learning curve of our methods when we increase the amount of training data, from 4 questions to the full training dataset. The line represents the mean MAP over 10 runs, while the shaded area represents the standard deviation.

In Figure 1, we illustrate the performance of two architectures, siamese and concat, combined with the two training approaches, point-wise and pair-wise, on the WikiQA dataset. We observe that the concat architecture, both with point-wise and pair-wise training, gives a low performance with few questions and the curve steeply rises. The siamese architecture starts at a rather high performace level, especially with the pair-wise learning scheme. The curve is rather flat compared to the one obtained with the concat architecture. This is most likely due to a lower number of parameters compared to the concat architecture and a fixed similarity function. Overall, the pair-wise training gives better performance than the point-wise one. We note that with the full training set the pair-wise/siamese and point-wise/concat give similar performance. We observe the same trends on the other datasets, except on Semeval.

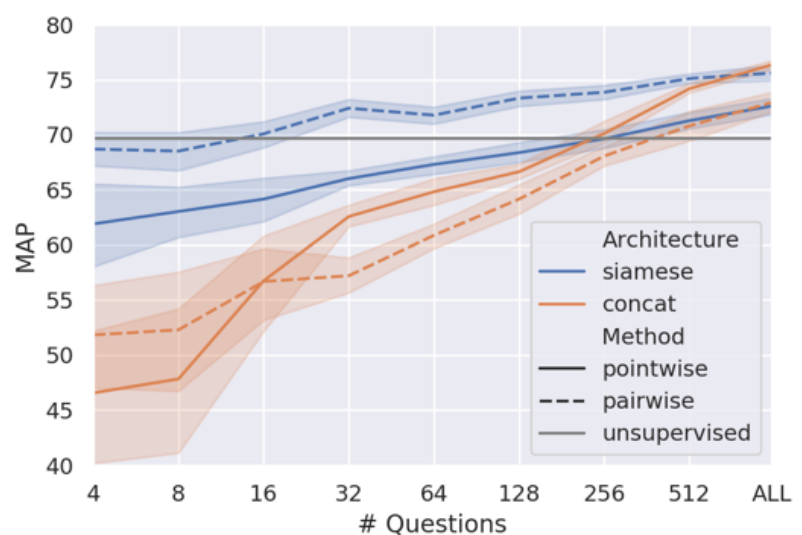

Figure 1: Comparison of the architectures and training approaches - MAP obtained on the WikiQA dataset when training the model on an increasing number of questions. We compared the two architectures, siamese and concat, as well as the two training approaches, point-wise and pair-wise. The grey line represents the MAP obtained with the unsupervised USE method.

For the latter, the concat architecture performs better, and all the approaches or architectures are above the unsupervised baseline for any number of questions.

In Figure 2, we show the effect of the pre-training on the performance on the WikiQA, Semeval, and Yahoo datasets. We do not display the results on the TrecQA dataset for a matter of clarity, but we observe a behaviour that is similar to that on the WikiQA dataset. Illustrated results are obtained with the pair-wise/siamese approach.

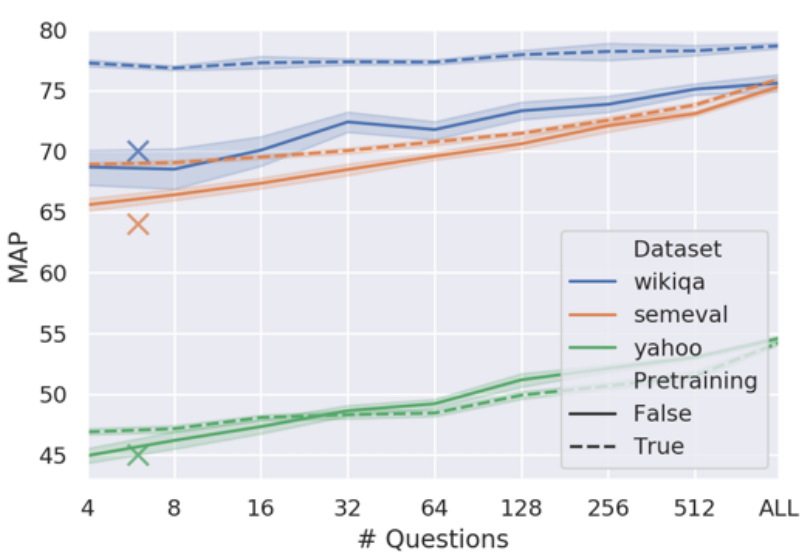

Figure 2: Effect of the pre-training - MAP obtained on the WikiQA, Semeval, and Yahoo datasets when training the pair-wise/siamese approach with USE representation on an increasing number of questions. We asses the gain obtained when pre-training the model on SQUAD-T. The crosses indicate the MAP with the unsupervised USE method (cosine).

Pre-training is highly effective on the WikiQA, Semeval (and TrecQA) datasets when training is performed on a 
few questions. The MAP on WikiQA remains constant up to a hundred questions, and then slowly increases. In the case of Semeval, pre-training gives a significant improvement of the performance for a small training set (up to 64). The gain in performance then gradually decreases, and it is close to zero when all the training set is used. For the Yahoo dataset, pre-training is only effective for a low number of training question (up to 16) and is detrimental when more questions are used for training. These findings confirm the added value of domain adaptation when the domain shift is not too important, i.e., for WikiQA and TrecQA. It is also efficient for the other datasets, but only when the number of training samples is very low, i.e., a few dozens.

Importantly, we note that the siamese architecture is very effective at learning a representation that outperforms the unsupervised baseline (shown with a cross). For all the datasets, the model learns a better representation than the baseline with as little as four questions.

\subsection{All labels in the target dataset}

In Table 4, we report the results obtained with the full training set. We show the concat architecture coupled with the point-wise training and the siamese architecture coupled with the pair-wise approach as they prove to be the best performing combinations.

Table 4: Results obtained using the full training set with the pointwise/concat architecture, with and without pre-training on SQUAD-T and with the $w 2 v$ or USE representation. + indicates that the method used SQuAD-T dataset for pre-training the model. * indicates that our best result is significantly different from our second best result for a given dataset. The SOTA 1 and 2 refers repectively to [9] and [10] for WikiQA, [23] and [9] for Semeval, [24] and [25] for TrecQA, and [26] and [6] for Yahoo.

\begin{tabular}{lllll}
\hline \multirow{2}{*}{ Method - Representation } & \multicolumn{3}{l}{ WikiQA } & \multicolumn{2}{l}{ TrecQA } & \multicolumn{2}{l}{ SemEvalYahoo } \\
& MAP & MAP & MAP & P@1 \\
\hline point/concat -w2v & 60.11 & 73.23 & 72.10 & 27.50 \\
point/concat -w2v $\dagger$ & 66.14 & $\mathbf{7 6 . 6 0 *}$ & 73.43 & 27.30 \\
point/concat - USE & 76.36 & 65.37 & $\mathbf{8 0 . 5 7 *}$ & $\mathbf{3 6 . 6 5 *}$ \\
point/concat - USE $\dagger$ & 78.30 & 72.52 & 80.45 & 34.87 \\
pair/siamese - w2v & 62.38 & 69.12 & 71.75 & 24.92 \\
pair/siamese - w2v $\dagger$ & 67.94 & 70.27 & 71.72 & 25.76 \\
pair/siamese - USE & 75.61 & 67.96 & 75.32 & 34.13 \\
pair/siamese - USE $\dagger$ & $\mathbf{7 8 . 6 7 *}$ & 71.65 & 75.94 & 33.79 \\
\hline SOTA 1 & $\mathbf{7 9 . 9 0}$ & $\mathbf{8 6 . 5 7}$ & 80.36 & $\mathbf{3 8 . 7 4}$ \\
SOTA 2 & 74.50 & 82.20 & $78.37 \dagger$ & 37.17 \\
\hline
\end{tabular}

On the WikiQA dataset, we obtain very good results, with a MAP close to the state-of-the-art which is an attentionbased RNN architecture also pre-trained on SQuAD-T [9]. Without pre-training, we obtain a MAP of 76.36 , which is superior to the state of the art without pre-training (74.50 MAP [10]).

On the Semeval dataset, the pre-training has a small, yet significant, effect on the performance, and our results are over the current state of the art.

Results on the TrecQA dataset exhibit a different pattern, as the w2v representation is better than the USE one. However, the performance reached by our approach is 10 points below the state of the art. The MAP is slightly, yet significantly, higher than with the unsupervised Hungarian method.

On the Yahoo dataset, we reach good performance with the USE representation, only 2 points below the state of the art. For this dataset, the pre-training is significantly detrimental $(-1.4$ points $p>0.05)$.

Overall, the best results are obtained with the pointwise/concat method, as the pair-wise/siamese approach only performs best on the WikiQA dataet, and with a small margin (0.3 points).

\section{Discussion}

In this paper, we explored several approaches for the answer selection task on four datasets and with a varying amount of labeled examples. We provided guidelines on the method to choose depending on the availability of annotations and external resources.

We designed a new unsupervised approach (Hungarian) that remains the safest choice when no annotation or external dataset are available. When an external dataset is available, the distantly supervised approach is better than the Hungarian method on two datasets out of four. Notably, the distantly supervised approach provides state-ofthe-art results on the WikiQA dataset, which is a remarkable result, given that no annotation were used on the target dataset.

Importantly, we found that the pair-wise/siamese method allows for low-shot learning of sentence representations that outperforms the unsupervised baseline with as little as four questions.

Pre-training the models on an external dataset and performing fine-tuning on the target dataset only provides good results when the latter does not present a domain shift, or when the number of training samples used for fine-tuning is low. We believe that performing a cross- 
domain regularization could greatly improve our results, using for instance an adversarial approach such as in [27]. We note that the siamese architecture would be of particular interest in the context of large-scale semantic information retrieval because the question and candidate answers are independently encoded. It results that a large answers database could be encoded offline and performing the search would just consist of very fast and efficient cosine operations [28].

Finally, we found that when a larger amount of data is available, our models compete with much more complex approaches typically relying on convolutional and/or recurrent models with attention mechanisms [2, 29]. We argue that the effectiveness of our methods relies on the use of pre-trained language model (USE) whereas other methods usually perform end-to-end training, which might not be appropriate for data with a limited number of annotated training samples.

\section{References}

[1] Rajpurkar P., Zhang J., Lopyrev K., Liang P., SQuAD: 100,000+ Questions for Machine Comprehension of Text, 2016, arXiv: 1606.05250 [cs]

[2] Yin W., Schütze H., Xiang B., Zhou B., ABCNN: Attention-Based Convolutional Neural Network for Modeling Sentence, Pairs, 2015, arXiv: 1512.05193 [cs]

[3] Wang S., Jiang J., A Compare-Aggregate Model for Matching Text Sequences, 2016, arXiv: 1611.01747 [cs]

[4] Tran N. K., Niedereée C., Multihop Attention Networks for Question Answer Matching, in The 41st International ACM SIGIR Conference on Research \& Development in Information Retrieval, SIGIR '18, ACM, New York, NY, USA, 2018, 325-334, 10.1145/3209978.3210009

[5] Shen D., Wang G., Wang W., Min M.R., Su Q., Zhang Y., Li C., Henao R., Carin L., Baseline Needs More Love: On Simple Word-Embedding-Based Models and Associated Pooling Mechanisms, arXiv:1805.09843 [cs], 2018, arXiv: 1805.09843 [cs]

[6] Bogdanova D., Foster J., This is how we do it: Answer Reranking for Open-domain How Questions with Paragraph Vectors and Minimal Feature Engineering, in HLT-NAACL, 2016

[7] Yadav V., Sharp R., Surdeanu M., Sanity Check: A Strong Alignment and Information Retrieval Baseline for Question Answering, 2018, arXiv: 1807.01836 [cs]

[8] Seo M., Kembhavi A., Farhadi A., Hajishirzi H., Bidirectional Attention Flow for Machine Comprehension, 2016, arXiv: 1611.01603 [cs]

[9] Min S., Seo M., Hajishirzi H., Question Answering through Transfer Learning from Large Fine-grained Supervision Data, 2017, arXiv: 1702.02171 [cs]

[10] Zhang P., Hou Y., Su Z., Su Y., Two-Step Multi-factor Attention Neural Network for Answer Selection, in PRICAI 2018: Trends in Artificial Intelligence, Springer, Cham, 2018, 658670, 10.1007/978-3-319-97304-3_50
[11] Kuhn H. W., The Hungarian method for the assignment problem, Naval Research Logistics Quarterly, 1955, 2(1-2), 83-97, $10.1002 /$ nav. 3800020109

[12] Mikolov T., Sutskever I., Chen K., Corrado G.S., Dean J., Distributed Representations of Words and Phrases and their Compositionality, in C.J.C. Burges, L. Bottou, M. Welling, Z. Ghahramani, K.Q. Weinberger, eds., Advances in Neural Information Processing Systems 26, Curran Associates, Inc., 2013, 31113119

[13] Cer D., Yang., Kong S. Y., Hua N., Limtiaco N., John R.S., Constant N., Guajardo-Cespedes M., Yuan S., Tar C., Sung Y.H., Strope B., Kurzweil R., Universal Sentence Encoder, 2018, arXiv: 1803.11175 [cs]

[14] Crane M., Questionable Answers in Question Answering Research: Reproducibility and Variability of Published Results, Transactions of the Association for Computational Linguistics, 2018, 6(0), 241-252

[15] Vaswani A., Shazeer N., Parmar N., Uszkoreit J., Jones L., Gomez A.N., Kaiser L., Polosukhin I., Attention Is All You Need, 2017, arXiv: 1706.03762 [cs]

[16] Robertson S. E., Jones K. S., Relevance weighting of search terms, Journal of the American Society for Information Science, 1976, 27(3), 129-146, 10.1002/asi.4630270302

[17] Lai T. M., Bui T., Li S., A Review on Deep Learning Techniques Applied to Answer Selection, in Proceedings of the 27th International Conference on Computational Linguistics, Association for Computational Linguistics, Santa Fe, New Mexico, USA, 2018, 2132-2144

[18] Wang M., Smith N. A., Mitamura T., What is the Jeopardy Model? A Quasi-Synchronous Grammar for QA, in Proceedings of the 2007 Joint Conference on Empirical Methods in Natural Language Processing and Computational Natural Language Learning (EMNLP-CoNLL), 2007

[19] Wang D., Nyberg E., A Long Short-Term Memory Model for Answer Sentence Selection in Question Answering, in Proceedings of the 53rd Annual Meeting of the Association for Computational Linguistics and the 7th International Joint Conference on Natural Language Processing (Volume 2: Short Papers), Association for Computational Linguistics, 2015, 707-712, 10.3115/v1/P152116, event-place: Beijing, China

[20] Nakov P., Màrquez L., Moschitti A., Magdy W., Mubarak H., Freihat a.A., Glass J., Randeree B., SemEval-2016 Task 3: Community Question Answering, in Proceedings of the 10th International Workshop on Semantic Evaluation, Association for Computational Linguistics, San Diego, California, 2016, 525-545, 10.18653/v1/S16-1083

[21] Jansen P., Surdeanu M., Clark P., Discourse Complements Lexical Semantics for Non-factoid Answer Reranking, in Proceedings of the 52nd Annual Meeting of the Association for Computational Linguistics (Volume 1: Long Papers), Association for Computational Linguistics, Baltimore, Maryland, 2014, 977-986, 10.3115/v1/P14-1092

[22] Kingma D.P., Ba J., Adam: A Method for Stochastic Optimization, 2014, arXiv: 1412.6980 [cs]

[23] Liu Y., Rong W., Xiong Z., Improved Text Matching by Enhancing Mutual Information, in AAAI, 2018

[24] Tayyar Madabushi H., Lee M., Barnden J., Integrating Question Classification and Deep Learning for improved Answer Selection, in Proceedings of the 27th International Conference on Computational Linguistics, Association for Computational Lin- 
guistics, Santa Fe, New Mexico, USA, 2018, 3283-3294

[25] Zhang H., Rao J., Lin J., Smucker M.D., Automatically Extracting High-Quality Negative Examples for Answer Selection in Question Answering, in Proceedings of the 40th International ACM SIGIR Conference on Research and Development in Information Retrieval, SIGIR '17, ACM, New York, NY, USA, 2017, 797-800

[26] Bogdanova D., Foster J., Dzendzik D., Liu Q., If You Can’t Beat Them Join Them: Handcrafted Features Complement Neural Nets for Non-Factoid Answer Reranking, in HLT-EACL, Association for Computational Linguistics, Valencia, Spain, 2017, 121-131
[27] Cohen D., Mitra B., Hofmann K., Croft W. B., Cross Domain Regularization for Neural Ranking Models Using Adversarial Learning, 2018, arXiv: 1805.03403 [cs]

[28] Seo M., Kwiatkowski T., Parikh A. P., Farhadi A., Hajishirzi H., Phrase-Indexed Question Answering: A New Challenge for Scalable Document Comprehension, 2018, arXiv: 1804.07726 [cs]

[29] Rücklé A., Gurevych I., Representation Learning for Answer Selection with LSTM-Based Importance Weighting, IWCS 2017 12th International Conference on Computational Semantics Short papers, 2017 